\title{
Congenital scoliosis treated with posterior vertebral column resection in patients younger than 18 years: longer than 10-year follow-up
}

\author{
*Dong-Gune Chang, MD, ${ }^{1}$ Jae Hyuk Yang, MD, ${ }^{2}$ Jung-Hee Lee, MD, ${ }^{3}$ Jin-Hyok Kim, MD, ${ }^{1}$ \\ Seung-Woo Suh, MD, ${ }^{2}$ Kee-Yong Ha, MD, ${ }^{4}$ and Se-II Suk, MD ${ }^{1}$ \\ 1Department of Orthopaedic Surgery, Sanggye Paik Hospital, Inje University; 2Department of Orthopaedic Surgery, \\ Guro-Hospital, Korea University; ${ }^{3}$ Department of Orthopaedic Surgery, Kyung Hee Hospital, Kyung Hee University; and \\ ${ }^{4}$ Department of Orthopaedic Surgery, Seoul St. Mary's Hospital, Catholic University of Korea, Seoul, Korea
}

\begin{abstract}
OBJECTIVE There have been no reports on the long-term radiographic outcomes of posterior vertebral column resection (PVCR) in patients with congenital scoliosis. The purpose of this study was to evaluate the surgical outcomes and complications after PVCR and its long-term effects on correcting this deformity in children with congenital scoliosis.

METHODS The authors retrospectively analyzed the medical records of 45 patients with congenital scoliosis who were younger than 18 years at the time of surgery and who underwent PVCR and fusion with pedicle screw fixation (PSF). The mean age of the patients at the time of surgery was 11.3 years (range 2.4-18.0 years), and the mean length of follow-up was 12.8 years (range 10.1-18.2 years).

RESULTS The mean Cobb angle of the main curve was $46.5^{\circ}$ before PVCR, $13.7^{\circ}$ immediately after PVCR, and $17.6^{\circ}$ at the last follow-up. For the compensatory cranial curve, PVCR corrected the preoperative Cobb angle of $21.2^{\circ}$ to $9.1^{\circ}$ postoperatively and maintained it at $10.9^{\circ}$ at the last follow-up. For the compensatory caudal curve, the preoperative Cobb angle of $23.8^{\circ}$ improved to $7.7^{\circ}$ postoperatively and was $9.8^{\circ}$ at the last follow-up. The authors noted 22 complications, and the overall incidence of complications was $48.9 \%$.

CONCLUSIONS Posterior vertebral column resection is an effective procedure for managing congenital scoliosis in patients younger than 18 years. Use of PVCR and fusion with PSF for congenital scoliosis achieved rigid fixation and satisfactory deformity correction that was maintained over the long term. However, the authors note that PVCR is a technically demanding procedure and entails risks for major complications and excessive blood loss.

http://thejns.org/doi/abs/10.3171/2015.11.SPINE151099
\end{abstract}

KEY WORDS congenital scoliosis; hemivertebra; posterior vertebral column resection; hemivertebra resection; pedicle screw fixation; long-term follow-up; spinal disorders

$\mathrm{C}$ ONGENITAL scoliosis results from abnormal formation or segmentation of vertebral elements leading to asymmetrical growth of the spine. ${ }^{23}$ A complete unilateral failure of vertebra formation causing only one side of the vertebral body to develop creates a hemivertebra, which is the most frequent cause of congenital scoliosis. ${ }^{12,25,28,34}$ Surgery is usually recommended for patients with severe spinal deformity, but the surgical treatment for severe spinal deformities in children is extremely challenging.

Surgical treatment for such patients may include 1) in situ fusion, 2) anterior or posterior fusion with or without instrumentation, 3) combined anterior and posterior convex hemiepiphysiodesis or hemiarthrodesis, 4) hemivertebra excision, or 5) posterior vertebral column resection (PVCR) and fusion. 1,7, ,13,14,18,20-22,26,30-33,35-40 The curative treatment of congenital scoliosis due to hemivertebra should include removal of the hemivertebra. Recently, successful posterior hemivertebra excision and PVCR in growing children have been reported. ${ }^{79}$

Posterior vertebral column resection was first reported by Suk et al. ${ }^{37}$ and has been popularized by Lenke et

ABBREVIATIONS CB = coronal balance; $L L=$ lumbar lordosis; MEP = motor evoked potential; $P$ VCR = posterior vertebral column resection; PSF = pedicle screw fixation; $\mathrm{SB}=$ sagittal balance; SK = segmental kyphosis; SSEP = somatosensory evoked potential; TK = thoracic kyphosis.

SUBMITTED September 15, 2015. ACCEPTED November 23, 2015.

INCLUDE WHEN CITING Published online March 11, 2016; DOI: 10.3171/2015.11.SPINE151099.

* Drs. Chang and Yang contributed equally to this work. 
al. ${ }^{20-22}$ for correcting severe spinal deformities. The technique enables translational and rotational correction of the spinal column and offers the advantage of a controlled manipulation of both anterior and posterior columns at the same time through a single posterior approach. ${ }^{30}$

Pedicle subtraction osteotomies (e.g., hemivertebra excision) remove the pedicle and vertebral body (with the hemivertebra) with no contact with the discs above and below the removed vertebral elements. In contrast, PVCR resects not only the pedicle and vertebral body with the hemivertebra but also the discs above and below the hemivertebra and results in body-to-body contact.,9,36-38 Posterior vertebral column resection can correct more deformities, particularly kyphotic deformity, through sufficient shortening of the posterior column and achieve increased bony union through bone-to-bone contact of the vertebral body even if the coronal curves are not severe. Therefore, PVCR is a very powerful operative method for correcting severe spinal deformities.

No reports in the literature exist that have described the long-term outcomes after posterior-only approaches involving PVCR to correct congenital scoliosis. We evaluated the outcomes of and complications arising from PVCR for congenital scoliosis in children younger than 18 years and the long-term effects of this technique on deformity correction, including curve maintenance.

\section{Methods \\ Patient Cohort}

The records of 66 patients who had a diagnosis of congenital scoliosis due to a hemivertebra and who underwent PVCR and posterior fusion with pedicle screw fixation (PSF) at our institution between 1997 and 2004 were retrospectively reviewed for deformity correction immediately after surgery and at long-term follow-up examinations. Our hospital institutional review board approved this study before data collection and analysis. The inclusion criteria were as follows: 1) congenital spinal defor- mity requiring surgical treatment for a curve magnitude greater than $25^{\circ}$ with fast progression, which included documented progression of the curve of more than $5^{\circ}$ in a 6-month period, failure of conservative treatment, or both; 2) PVCR with bilateral pedicle screw instrumentation; 3) age at the time of surgery younger than 18 years (the skeleton development tends to stop at this age); and 4) a minimum follow-up length of 10 years.

All of the patients in this study underwent PVCR (see Fig. 1 for an example). Cases that involved simple excision of the hemivertebra, pedicle, or both, or of a vertebral body without disc excision, were excluded from this study.

The clinical and radiographic follow-up evaluations were conducted postoperatively (within 4 weeks) and at 3 months, 6 months, 1 year, 2 years, and then every 2-3 years thereafter. Of 66 patients, 20 were lost to follow-up, and 1 patient was excluded because of a vague margin of anatomical markers. Therefore, 45 patients met our criteria, and the 10-year follow-up rate was $68.2 \%$ (45 of 66 patients). All patients were younger than 18 years at the time of the operation, with a mean age of 11.3 years (range 2.4-18.0 years); the mean length of the follow-up period was 12.8 years (Table 1$)$.

The patient cohort included 29 boys and 16 girls. Of these 45 patients, 10 showed additional anomalies in the following structures or organs: 1) spine (4 patients; 1 astrocytoma and 3 syringohydromyelia), 2) genitourinary system (4 patients; 2 ectopic kidneys, 1 renal agenesis, and 1 corpus luteal cyst), and 3) cardiopulmonary system (2 patients; 1 Tetralogy of Fallot and 1 tricuspid valve regurgitation).

Twenty-four of the patients had a single-level hemivertebra, and 21 had complex congenital deformities, which included double hemivertebrae in 4 patients, multiple hemivertebrae in 11 patients, hemivertebra with a block vertebra in 2 patients, and hemivertebra with unsegmented bar in 4 patients (Table 2). Fifty-four PVCR operations were performed; 11 patients underwent PVCR in the tho-
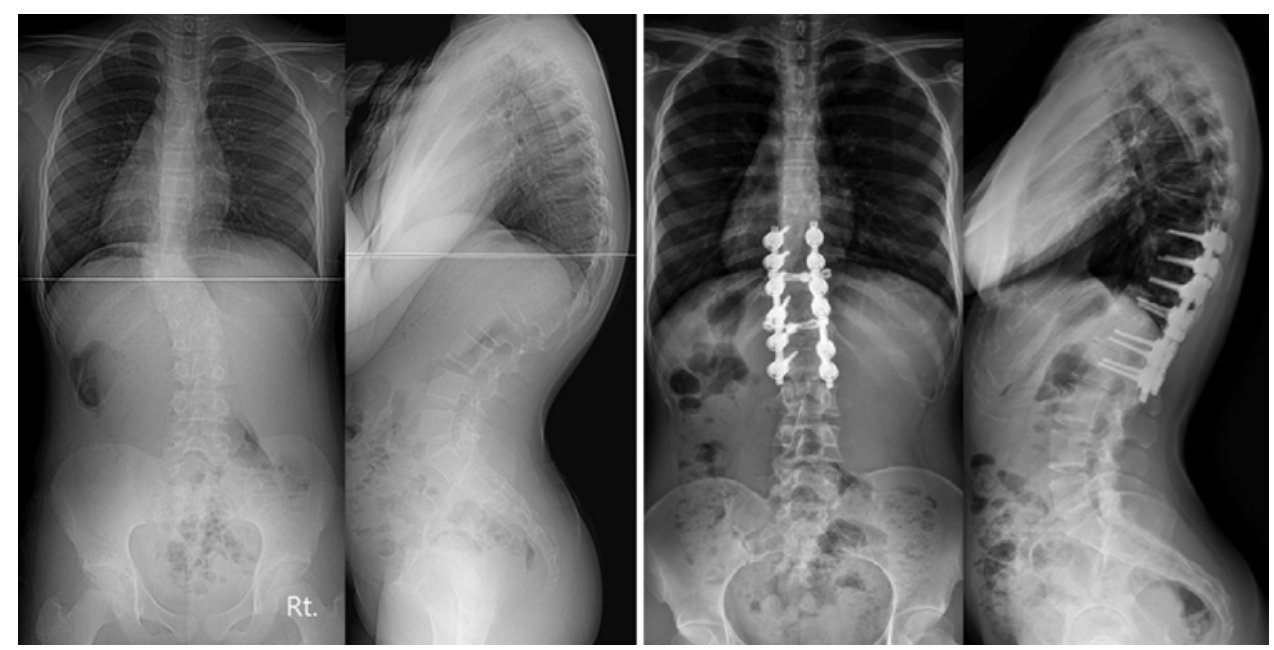

FIG. 1. Left: Dorsoventral and lateral radiographs of the spine of a 10-year-old girl who had a congenital hemivertebra at T-11 and whose spine had a left thoracolumbar scoliosis angle of $40^{\circ}$ and an SK angle of $54^{\circ}$. The patient underwent PVCR of T- 11 and posterior T9-L2 PSF. Right: Dorsoventral and lateral 11-year follow-up radiographs indicating that the main curve showed no progression and maintained an angle of $12^{\circ}$. 
TABLE 1. Demographic and operative data*

\begin{tabular}{lc}
\hline \multicolumn{1}{c}{ Demographic \& Op Variables } & Value \\
\hline No. of patients & 45 \\
\hline M/F ratio & $29: 16$ \\
\hline Age at the time of op in yrs & $11.3(2.4-18.0)$ \\
\hline Length of FU in yrs & $12.8(10.1-18.2)$ \\
\hline No. of fused segments & $4(1-12)$ \\
\hline No. of cages used for ant support & 4 \\
\hline Op time in mins & $221(80-405)$ \\
\hline EBL in ml & $1916(270-6000)$ \\
\hline
\end{tabular}

Ant = anterior; $E B L=$ estimated blood loss; $F U$ = follow-up

* Data represent the mean (range), unless indicated otherwise.

racic spine (T1-9), 34 in the thoracolumbar region (T10L2), and 9 in the lumbar spine (L3-5).

\section{Surgical Procedures}

The senior author performed all surgeries, which were conducted with somatosensory evoked potential (SSEP) monitoring. The posterior elements of the spine were carefully exposed at the affected and adjacent levels, including the lamina, facet joint, and transverse processes.

In cases of thoracic hemivertebrae, the rib heads and costotransverse joints on the convex side were removed to allow for complete resection of the lateral wall of the vertebral body and for untethered motion of the vertebral column. In the lumbar spine, the transverse process was osteotomized at its base and left there with muscle attached to facilitate bony union. Facetectomy was performed to promote intraarticular fusion and soft-tissue release; pedicle long-arm reduction screws were inserted segmentally on both sides, except for the resected hemivertebra. Intraoperative posteroanterior and lateral radiographs with $\mathrm{K}$-wires at the presumed ideal pedicle entry points were taken to monitor safe insertion of the pedicle screws.

The neural elements, including the dura and nerve roots, were identified with wide laminectomy and bilateral foraminotomies. After the screws on the concave side of the spinal curve were fixed with a temporary rod, meticulous subperiosteal dissection was performed down to the lateral wall of the vertebral body of the hemivertebra until the anterior surface of the body was easily palpable. Segmental vessels were carefully preserved. Under direct vision, the pedicles and vertebral body of the hemivertebra were removed with a small osteotome and rongeur.

The hemivertebra body and the adjacent discs on both sides above and below it were removed in arbitrary order and gradually toward the medial and opposite sides. A thin rim of the posterior vertebral wall beneath the dura was left intact. The anterior wall was also removed in this fashion, and care was taken to leave the soft tissue tube anterior to the vertebral body intact. When an adequate amount of vertebral body was removed, the entire portion of the posterior wall was removed with an Epstein reversecutting curette and pituitary forceps. After the resection of the posterior wall on the convex side, another temporary rod was inserted on this side and securely locked to the
TABLE 2. Etiology of the spinal disorders

\begin{tabular}{lc}
\hline \multicolumn{1}{c}{ Etiology } & No. of Patients \\
\hline Single hemivertebra & 24 \\
\hline Double hemivertebrae & 4 \\
\hline Multiple hemivertebrae & 11 \\
\hline Hemivertebra w/ block vertebra & 2 \\
\hline Hemivertebra w/ unsegmented bars & 4 \\
\hline
\end{tabular}

screws with slight compression to shorten the vertebral column. The temporary rod on the concave side was then removed to allow discectomy and resection of the remaining hemivertebra.

Because in a hemivertebra there is no pedicle or vertebral body on the concave side, both the upper and lower discs of the hemivertebra on this side are merged and form one disc. This concave disc remnant had to be removed for complete release of the deformity. The deformity was gradually corrected by repeated additional compression and shortening of the vertebral column, which was compressed by alternately tightening down the temporary rods on both sides. The compression causing a shortening of the resected gap was continued until there was an excess of exposed dura without unintended distraction or kinking of the neural elements. After compression and shortening of the resected gap, the temporary rods were replaced one by one with precontoured permanent rods on both sides to avoid any loss of correction at the resected gap. Arthrodesis by posterior fusion was carried out with local bone after decortication.

\section{Postoperative Care}

Patients underwent mobility exercises at 1 week postoperatively in a localizer cast, which was worn for 3 months, and were then fitted with a thoracolumbosacral orthosis for 3-6 months to maintain spinal stability, correct the deformity, enhance the bony union, and allow early mobilization.

\section{Radiographic Measurements}

Whole-spine anteroposterior and lateral radiographs were reviewed preoperatively, 4 weeks postoperatively, and at the last follow-up to assess the deformity correction and spinal balance.

The magnitudes of the spinal curves were assessed with several parameters. Coronal balance (CB), sagittal balance (SB), segmental kyphosis (SK), thoracic kyphosis (TK), and lumbar lordosis (LL) were measured as balance parameters. ${ }^{4,5,7-9,16,29}$ The magnitudes of both main and compensatory curves were measured with the Cobb method, using the end vertebrae determined on the preoperative standing radiographs. Coronal balance and SB were measured on standing radiographs; $\mathrm{CB}$ was measured as the deviation of the C-7 plumb line from the center sacral vertical line and SB as the distance from the C-7 plumb line to a perpendicular line drawn from the posterosuperior corner of the sacrum. Sagittal balance was assessed positive (+) when the $\mathrm{C}-7$ plumb line was anterior to the 
posterosuperior corner of the sacrum and negative (-) when the C-7 plumb line was posterior to the posterosuperior corner of the sacrum.

Segmental angles of kyphosis or lordosis were measured from the upper endplate above the hemivertebra to the lower endplate below the hemivertebra. Lumbar lordosis was measured from the upper endplate of T-12 to the upper endplate of the sacrum. The TK was measured from the upper endplate of T-5 to the lower endplate of T- 12 .

To minimize interobserver measurement error, all radiographs were examined by 2 authors who did not participate in the operation. The means of these measurements were used in the analysis.

\section{Statistical Analysis}

A repeated-measures ANOVA test was performed for comparisons among dependent variables. Student's t-test was performed at each time point as a within-subject variable. A difference was considered statistically significant at $\mathrm{p}<0.05$.

\section{Results}

\section{Deformity Correction}

For all patients, the mean preoperative Cobb angle of the main curve of $46.5^{\circ}$ improved to $13.7^{\circ}$ immediately after PVCR and was $17.6^{\circ}$ at the last follow-up. The mean correction rate was $70.5 \%$ immediately postoperatively and $62.2 \%$ at the last follow-up, indicating a mean loss of correction of $8.3 \%$ during the follow-up period (Table 3 ).

For the compensatory cranial curve, the mean preoperative Cobb angle of $21.2^{\circ}$ improved to $9.1^{\circ}$ postoperatively and was $10.9^{\circ}$ at the last follow-up. The correction rate was $57.1 \%$ postoperatively and $48.6 \%$ at the last follow-up, indicating a loss of correction of $8.5 \%$ during the followup period.

For the compensatory caudal curve, the mean preoperative Cobb angle of $23.8^{\circ}$ improved to $7.7^{\circ}$ postoperatively and was $9.8^{\circ}$ at the last follow-up. The correction rate was $67.6 \%$ postoperatively and $58.8 \%$ at the last follow-up, indicating a loss of correction of $8.8 \%$ during the follow-up period. With the PVCR procedure, correction of the compensatory cranial curve was less than that for the compensatory caudal curve both immediately postoperatively and at the last follow-up (Fig. 2).

\section{Changes in CB and SB}

The mean preoperative $\mathrm{CB}$ of $11.5 \mathrm{~mm}$ improved to $9.6 \mathrm{~mm}$ immediately postoperatively and was $9.1 \mathrm{~mm}$ at the last follow-up. The mean preoperative SB of $-5.3 \mathrm{~mm}$ improved to $2 \mathrm{~mm}$ postoperatively and was $19.7 \mathrm{~mm}$ at the last follow-up (Table 4). However, these changes were not statistically significant, and CB was less influenced by the procedure than SB. The CB was maintained during the follow-up period, but SB deteriorated during that time.

\section{Changes in the Sagittal Plane}

All hemivertebra spines showed kyphotic deformity (i.e., a positive value) before PVCR. The mean Cobb angle of SK in all patients was $31.6^{\circ}$ preoperatively and signifi-
TABLE 3. Changes in curve angles after PVCR

\begin{tabular}{lccc}
\hline & \multicolumn{3}{c}{ Mean Angle \pm SD $\left(^{\circ}\right)$} \\
\cline { 2 - 4 } \multicolumn{1}{c}{ Spinal Curve } & Preop & $\begin{array}{c}\text { Immediately* } \\
\text { Postop }\end{array}$ & At Last FU* \\
\hline Main & $46.5 \pm 15.9$ & $13.7 \pm 8.0(70.5) \dagger$ & $17.6 \pm 8.4(62.2)$ \\
\hline $\begin{array}{l}\text { Compensatory } \\
\text { cranial }\end{array}$ & $21.2 \pm 12.9$ & $9.1 \pm 7.3(57.1) \dagger$ & $10.9 \pm 7.5(48.6)$ \\
\hline $\begin{array}{l}\text { Compensatory } \\
\text { caudal }\end{array}$ & $23.8 \pm 10.9$ & $7.7 \pm 5.8(67.6) \dagger$ & $9.8 \pm 7.4(58.8)$ \\
\hline * Values in parentheses represent the percentage change in angle from that \\
$\begin{array}{l}\text { preoperatively. } \\
\dagger \text { Statistically significantly }(p<0.05) \text { change from preoperative angle. }\end{array}$
\end{tabular}

cantly improved to $8.7^{\circ}$ immediately postoperatively and was $7.7^{\circ}$ at the last follow-up. The mean Cobb angle of TK was $24.5^{\circ}$ before surgery, $21.5^{\circ}$ immediately postoperatively, and $24.5^{\circ}$ at the last follow-up. The mean LL angle was $42.7^{\circ}$ before surgery, $37.2^{\circ}$ immediately postoperatively, and $41.3^{\circ}$ at the last follow-up (Table 4). Consistent with previous reports, PVCR for hemivertebra correction did not significantly influence TK and LL because of the proximal and distal curve compensations at the segments adjacent to the PVCR sites. ${ }^{31}$

\section{Operative Data}

As shown in Table 1, the mean operative time was 221 minutes (range 80-405 minutes), and the estimated blood loss was $1916 \mathrm{ml}$ (range 270-6000 ml).

\section{Complications}

We observed 22 complications in 16 patients (Table 5), and the overall complication incidence was $48.9 \%$. We noted 4 cases of transient neurological deficits immediately postoperatively that resolved within 3 months. Two of these deficits were caused by hematomas, which were evacuated immediately. One case of superficial wound infection was treated with incision and drainage. There was 1 case of screw malposition, but no major vascular or permanent neurological complications related to the pedicle screws inserted were observed.

Several late postoperative complications occurred as follows. In 4 patients, we observed screw pullout or metal failure, which consisted of a broken rod with distal screw pullout, and 2 of these patients underwent revision surgery. Five patients showed adding-on deformity with a progression of the curve. Of these 5 patients, 2 were fitted with a brace (for an example, see Fig. 3), and 3 underwent revision surgery (for an example, see Fig. 4). In 2 patients, late wound infections occurred that were addressed by removing the implant. None of the patients showed the crankshaft phenomenon at the last follow-up.

\section{Discussion}

The treatment of patients with severe pediatric deformities has traditionally included both anterior and posterior approaches to the spinal column. This is still the most common treatment for patients with severe and rigid spinal deformities. Recently, vertebral resection performed 

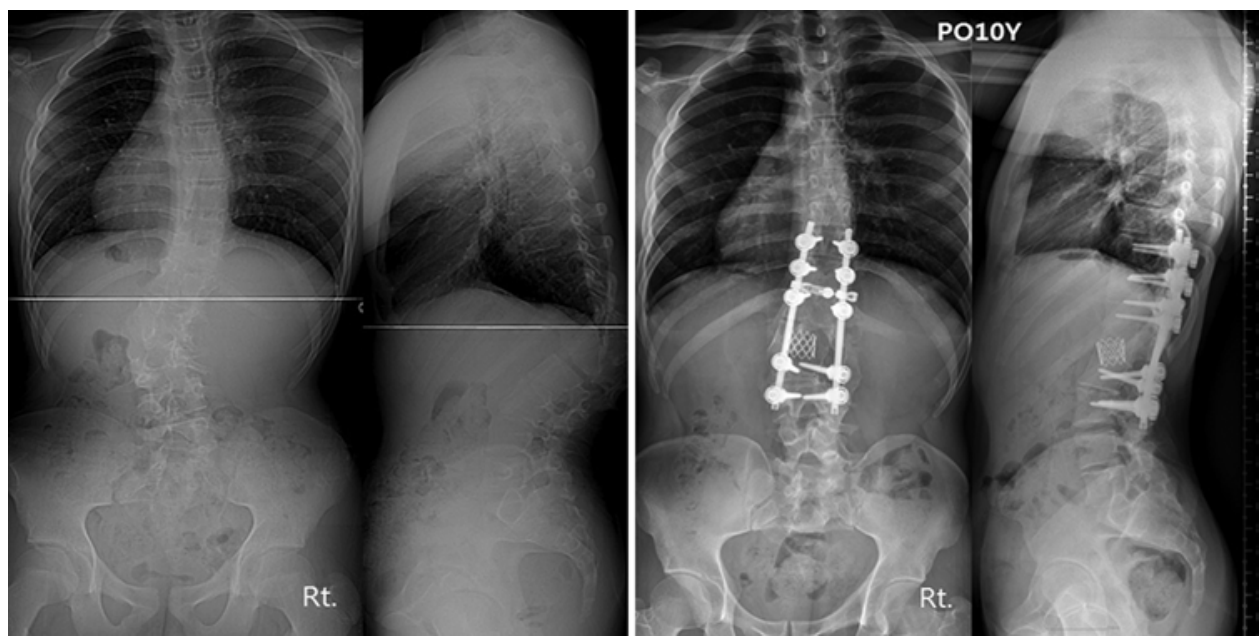

FIG. 2. Left: Dorsoventral and lateral radiographs of the spine of a 14-year-old-boy who had congenital hemivertebra at T-12 and $\mathrm{L}-1$ and whose spine had a left thoracolumbar scoliosis angle of $50^{\circ}$ and an SK angle of $52^{\circ}$. The patient underwent PVCR of L-1 and posterior fusion of T9-L3 with pedicle screws and received anterior support with a mesh cage. Right: Dorsoventral and lateral 10-year follow-up (PO10Y) radiographs indicating that the main curve in this patient showed no progression, and the scoliosis was well maintained at an angle of $18^{\circ}$ and an SK of $10^{\circ}$.

either circumferentially or via a posterior-only approach using PSF has become an alternative treatment for these spinal deformities. . $, 3,10,17,20-22,35-37^{2}$

Vertebral column resection for rigid scoliosis was first described by MacLennan in 1922. ${ }^{24}$ This procedure was performed through a costotransversectomy approach. Since this first report, many authors have noted that vertebral column resection is effective in treating patients with rigid spinal deformities. For instance, Leatherman reported performing a circumferential vertebral column resection for severe rigid spinal deformity..$^{19}$ In the late 1980s, Bradford reported on the use of a circumferential vertebral column resection coupled with concave rib osteotomies, convex thoracoplasty, and segmental spinal instrumentation with fusion in 13 patients. ${ }^{6}$ In 1991, Boachie-Adjei and Bradford expanded on Bradford's original case series by reporting on the outcomes in 16 patients who underwent a circumferential vertebral column resection, which resulted in excellent deformity correction. ${ }^{3}$

More recently, Suk et al. ${ }^{37}$ introduced a posterior-only approach to vertebral column resection for fixed lumbar

TABLE 4. Changes in coronal and sagittal balance and in Cobb angles of the operated spines*

\begin{tabular}{lccc}
\hline & \multicolumn{3}{c}{ Time Point } \\
\cline { 2 - 4 } Variable & Preop & Immediately Postop & Last FU \\
\hline $\mathrm{CB}(\mathrm{mm})$ & $11.5 \pm 9.3$ & $9.6 \pm 11.3$ & $9.1 \pm 7.1$ \\
\hline $\mathrm{SB}(\mathrm{mm})$ & $-5.3 \pm 25.8$ & $2.0 \pm 20.7$ & $19.7 \pm 22.3 \dagger$ \\
\hline $\mathrm{SK}\left(^{\circ}\right)$ & $31.6 \pm 18.7$ & $8.7 \pm 13.3 \dagger$ & $7.7 \pm 12.9$ \\
\hline $\mathrm{TK}\left(^{\circ}\right)$ & $24.5 \pm 17.6$ & $21.5 \pm 11.5$ & $24.5 \pm 9.5$ \\
\hline $\mathrm{LL}\left({ }^{\circ}\right)$ & $42.7 \pm 19.3$ & $37.2 \pm 13.3$ & $41.3 \pm 13.5$ \\
\hline
\end{tabular}

* Data represent mean \pm SD.

$\dagger$ Statistically significant $(p<0.05)$ change from angle at the previous time point. deformities and severely rigid scoliosis. ${ }^{35,36}$ Many authors also have reported on sequential series of PVCR. For example, Lenke et al. noted that PVCR improved the major curve in 35 children $(51 \%$ with scoliosis, $55 \%$ with global kyphosis, 58\% with angular kyphosis, 54\% with kyphoscoliosis, and $60 \%$ with congenital scoliosis), whose deformities were due to congenital, idiopathic, or neuromuscular causes, but these authors also observed a $40 \%$ overall complication rate with this technique..$^{20-22}$

Posterior vertebral column resection in young children is not yet well established. To the best of our knowledge, until now, there have been no reports on surgical outcomes of PVCR in the treatment of congenital scoliosis with long-term follow-up (i.e., longer than 10 years). Therefore, we focused on the deformity correction after PVCR and its long-term effects, including curve maintenance as well

\section{TABLE 5. Observed complications}

\begin{tabular}{cc}
\hline \multicolumn{1}{c}{ Complication } & No. of Occurrences \\
\hline Early complications & \\
\hline Transient neurological deficit & 4 \\
\hline Dural tear & 3 \\
\hline Hematoma & 2 \\
\hline Wound infection, superficial & 1 \\
\hline Wound infection, deep & 0 \\
\hline Screw malposition & 1 \\
\hline Major vascular injury & 0 \\
\hline Late complications & \\
\hline Permanent neurological deficit & 0 \\
\hline Metal failure/screw pullout & 4 \\
\hline Curve progression/adding-on & 5 \\
\hline Wound infection & 2 \\
\hline Crankshaft phenomenon & 0 \\
\hline
\end{tabular}



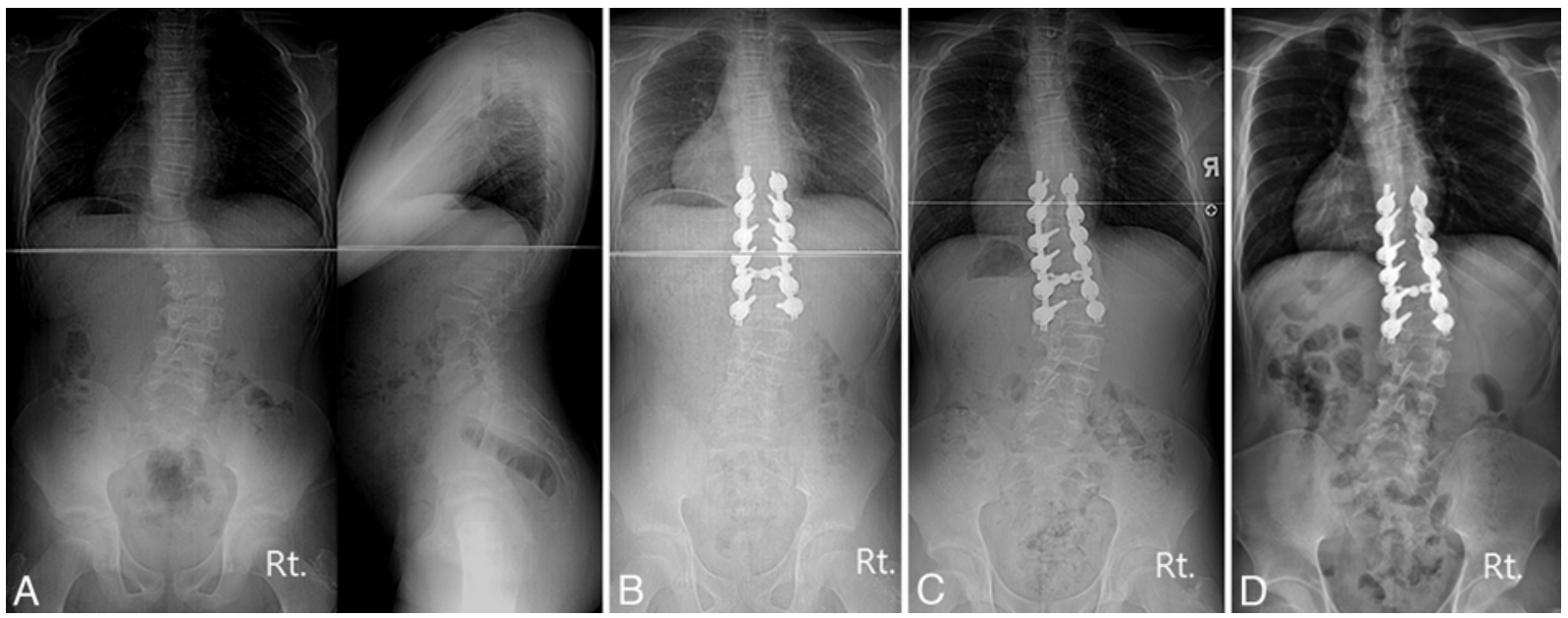

FIG. 3. A: Dorsoventral and lateral radiographs of the spine of a 13 -year-old boy who had congenital hemivertebra at T-11 and whose spine had a $41^{\circ}$ scoliosis angle and an SK angle of $27^{\circ}$. The patient underwent PVCR of T-11 and posterior fusion of T8-L2 with pedicle screws. B: A 9-month postoperative radiograph showing that the main curve improved to $5^{\circ}$ with satisfactory deformity correction. C: A 2-year follow-up radiograph demonstrating that the distal lumbar curve progressed, and this progression was managed with a brace. D: A 10-year follow-up radiograph of the same patient.

as complications in children younger than 18 years with congenital scoliosis.

The fusion level should be selected for correcting the deformity, preventing curve progression, and reducing the influence of adjacent vertebral growth. However, the fusion should be as short as possible. In addition, posterior instrumented fusion can lead to an abrupt transition from mobile segments to stiff junctional stress areas, extensive dissection of the paraspinal musculature, and resection of those lamina and facet joints that weaken the integrity of the posterior structures above and below the fusion segments. ${ }^{7,8}$ It is sufficient to use PVCR with monosegmental or 2-level fixation in children 6 years or younger, that is, before structural changes occur above and below the vertebral bodies resected by PVCR. In children of older age, fusion of more than 2-3 levels above and below the PVCR is usually required because short fusion with PVCR may induce adding-on deformity when the levels are fused with segmental PSF.

In spite of the presence of complex and severe deformities in the patients in our study, we did not use any intraoperative spinal navigation systems. We evaluated the preoperative CT axial view and used the K-wire method during the pedicle screw insertion as follows. The presumed pedicle entry points were decorticated with a rongeur to facilitate the insertion of the guide pins. The guide pins were inserted at a depth of $1 \mathrm{~cm}$ through the exposed cancellous bone at the presumed pedicle entry point. Different sizes of K-wires were used on both sides.

Intraoperative posteroanterior and lateral roentgenograms were taken to determine the association between the presumed entry point and the ideal entry point identified on the radiograph. These radiographs helped determine the ideal direction of the screws. Taking the transverse angle of the pedicles into consideration, we defined the ideal pedicle entry point in a neutrally rotated vertebra at the junction of a line parallel to the vertebra endplates and bisecting the pedicle and the lateral margin of the pedicle ring shadow on the posteroanterior radiograph. We also considered that in the rotated vertebrae, the ideal pedicle entry point of the pedicles on the side of the rotation (i.e., the convex side of the scoliosis) moves more medially, whereas the ideal pedicle entry point on the opposite side (the concave side) moves more laterally with increasing vertebral rotation. ${ }^{35-38}$

According to the results of the present study, correction rates of the main curve with PVCR were similar to those reported in previous studies of the technique. A systematic review of PVCR for correcting spinal deformity by Yang et al. ${ }^{41}$ reported a scoliosis correction rate of the main curve of $61.2 \%$. Ozturk et al..$^{30}$ reported a main scoliotic curve correction of $62 \%$ for PVCR in the treatment of patients with severe congenital kyphosis, scoliosis, or kyphoscoliosis. Lenke et al. ${ }^{21}$ reported a correction rate of the major curve of $60 \%$ in pediatric patients with congenital scoliosis, and Jeszenszky et al. ${ }^{15}$ noted that PVCR corrected the major curve at a rate of $57 \%$ in patients with early-onset spinal deformities. Our main curve correction rates of $70.5 \%$ after surgery and $62.2 \%$ at the last followup were similar to but somewhat higher than those observed in these previous reports, which may have included patients with much more severe preoperative deformities. We note that the results of our study, based on a follow-up period of more than 10 years, show that PVCR improved the main curve long term.

Curve corrections with posterior hemivertebra resection have been reported to range from $30 \%$ to $78 \%$ for the compensatory cranial curve and to reach about $60 \%$ for the compensatory caudal curve. ${ }^{27,31-33}$ In the present study, the correction rates of the compensatory cranial curve were $57.1 \%$ immediately postoperatively and $48.6 \%$ at the last follow-up, and those for the compensatory caudal curve were $67.6 \%$ immediately postoperatively and $58.8 \%$ at the last follow-up. This finding indicates that PVCR corrects the cranial compensatory curve to a lesser extent than the caudal compensatory curve. 

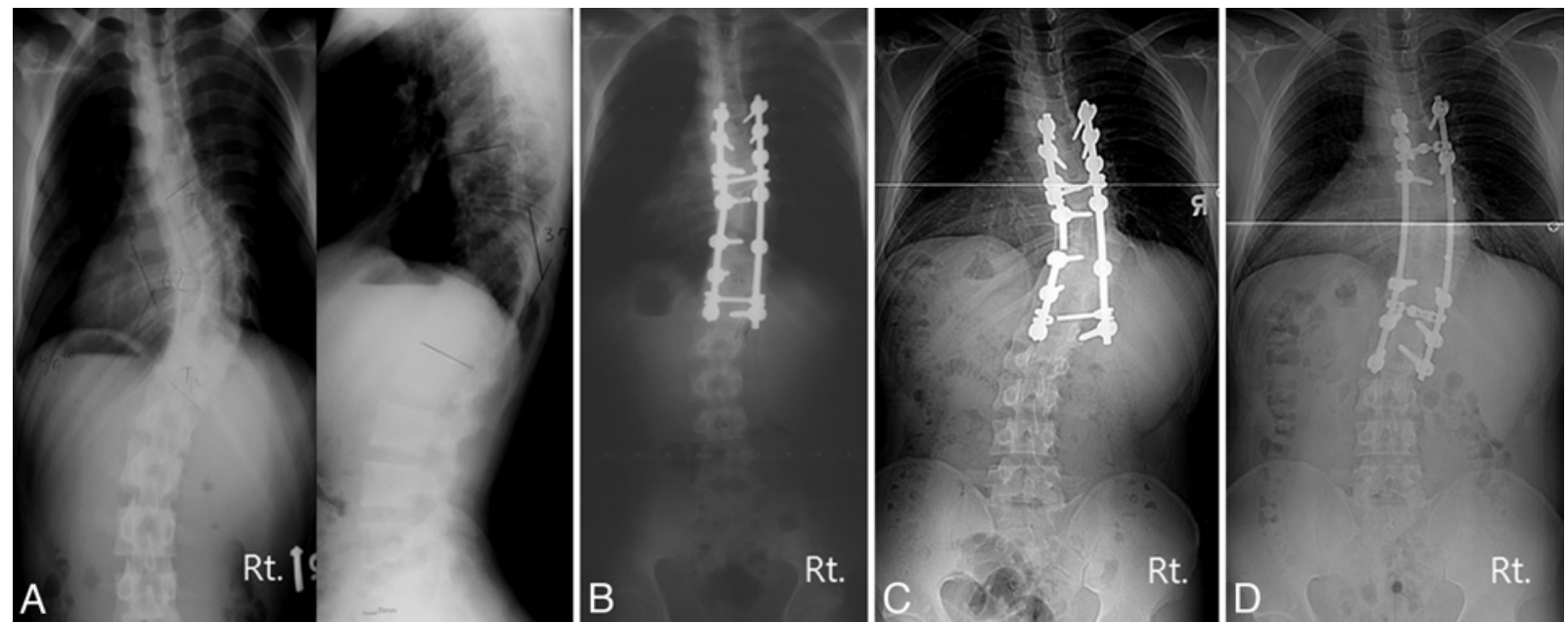

FIG. 4. A: Dorsoventral and lateral radiographs of the spine of an 18-year-old boy who had congenital scoliosis with a unilateral unsegmented bar at T8-10 and whose spine had a $62^{\circ}$ scoliosis angle and an SK angle of $37^{\circ}$. The patient underwent PVCR of T-10 and posterior fusion of T6-L1with pedicle screws. B: A 1-month postoperative radiograph showing that the main curve improved to $23^{\circ}$ with satisfactory deformity correction. C: A 6-year follow-up radiograph reveals metal failure (a broken rod). D: A 3-year follow-up radiograph after the patient underwent revision surgery for the instrumentation failure.

Coronal balance and SB were both not much affected by PVCR. Although CB was maintained at the last follow-up, SB became worse during the follow-up period. This observation indicates that SB may deteriorate during further growth, even if the SB was initially within the normal range for a child or adolescent. The $\mathrm{CB}$ and $\mathrm{SB}$ values varied between the serial radiographic assessments, especially in very young children, and depended on body position. Nevertheless, the spine was sufficiently balanced in most of the patients at the last follow-up. Regarding the sagittal alignments, both TK and LL values did not significantly change after PVCR and also remained stable during the long follow-up period. The values for TK and LL were less influenced by the PVCR for hemivertebra because of compensation within the adjacent segments. ${ }^{31}$

Anterior fusion across the resection gap was performed in all patients. The anterior column reconstruction was performed with autogenous cancellous bone grafts in all patients and additional titanium mesh was inserted in 4 patients. After resection and deformity correction, the height of the anterior interbody gap was measured, and if the height was less than $5 \mathrm{~mm}$, an autogenous cancellous chip bone was placed into the anterior gap. If the height was greater than $5 \mathrm{~mm}$, a titanium mesh cage and additional autogenous bone were placed to provide reliable anterior column reconstruction without excessive shortening. An optimal size of the anterior structural graft would allow a tight fit of the anterior gap, effectively supporting the load and increasing the chance of bony fusion without causing compression or undue tension in the neural elements. Titanium meshes have the advantage of offering additional stability because they are strong enough to be pushed into the bone and are serrated at both ends so that they can enter the bone beds through compression. This property provides structural support for load transfer from above to below the resected vertebrae during the period before bony fusion is complete. ${ }^{35-37}$
The estimated blood loss in our study was somewhat high for relatively short PVCR procedures in children. Reconstructive spinal surgeries may lead to a large blood loss, which is a primary potential cause for increased morbidity rates and postoperative complications. Accurate hemostasis is therefore critical. Although we did not administer antifibrinolytics intraoperatively, we meticulously performed hemostasis with electric cauterization and gauze packing and used only bone wax and hemostatic matrices (e.g., Gelfoam or Surgicel). Recently, we have begun administering antifibrinolytics (i.e., tranexamic acid) intraoperatively and using various types of hemostatic matrices for reducing blood loss during PVCR for treating patients with severe scoliosis because substantial bleeding in PVCR results from both the resections of the pedicles and vertebral body and the disruption of fragile epidural veins.

One major concern of PVCR is the risk for neurological complications. In this study, we observed 4 cases of transient neurological deficits, but none was permanent. This may be related to the fact that the spinal cord and nerve roots were under direct observation during the PVCR, and, therefore, direct injury could be avoided. Monitoring of motor evoked potentials (MEPs) was introduced in 2006 and was not available in our hospital at the time the patients in this study were operated on. We used SSEP monitoring or the wake-up test. We note that neuromonitoring (with MEPs) should now be imperative during PVCR procedures.

Pseudarthroses do not always result in instrumentation failures. We did not check CT scans routinely at the follow-ups for monitoring bony healing, but we used the scans if we suspected metal failures or pseudarthroses. We observed 4 cases of metal failure or screw pullout during the follow-up period, and CT scans were performed, but no additional pseudarthroses were detected in any of the patients at the last follow-up. Five of the patients showed the adding-on phenomenon with a progression of the curve. We noted 1 case of main curve progression and 
4 cases of compensatory curve progression. Of these 5 patients, 3 underwent revision surgery. The need for revision surgery for curve progression may be due to incomplete hemivertebra resection, improper fusion levels, or failure of anteroposterior fusion.

One patient underwent revision surgery for main curve progression. This progression may have been due to incomplete hemivertebra resection, improper fusion levels, or both. The remnant of the hemivertebra remaining after surgery may cause deterioration of the main curve and of the compensatory curves. Patients with progression of compensatory curves had no increased curvature at the PVCR levels that were fused with segmental PSF. This observation may be due to a short fusion, which may induce an increase in the curvature of more than $20^{\circ}$ above and below the fusion levels. We note that it is important that the adjacent compensatory curve be corrected to less than $20^{\circ}$.

The crankshaft phenomenon results from the continued growth of the anterior column in the presence of a posterior tether, which causes rotation of the fused segments. ${ }^{11}$ This mainly occurs in skeletally immature patients younger than 10 years who have open triradiate cartilages. We did not observe any cases of the crankshaft phenomenon. This adverse effect was probably prevented by the biomechanical characteristics of the pedicle screws that served as structural ties and provided resistance to the longitudinally directed forces through the 3 columns of the spinal vertebra. ${ }^{31,39}$

We have learned a lot from our extensive experience with PVCR and recommend the following practices to reduce postoperative complications with PVCR: 1) note that the level of resection should be the apex of the deformity; 2) use monoaxial, long-arm reduction screws, which provide rigid fixation, shorter fusion, easy insertion of the rod into screws, and gradual correction by compression alternately on both sides; 3 ) apply pedicle screws in every segment; 4) fix the temporary rod before destabilization; 5) do not use intraoperative rod bending in situ; 6) use a cage for anterior column support when the gap is more than 5 $\mathrm{mm}$ wide, and 7) perform neuromonitoring (with SSEPs or MEPs) at all times.

This study has some limitations. Data were analyzed retrospectively, and because the PVCR outcomes were evaluated only with radiographic parameters without any corresponding clinical parameters such as pain or disability scores, clinical information on the patients' quality of life or functional outcomes were not available. Therefore, additional studies are needed to correlate radiographic findings after PVCR with clinical outcomes (e.g., with Scoliosis Research Society 24, visual analog scale, or Oswestry Disability Index scores). Moreover, cosmesis plays an important role in the satisfaction of congenital scoliosis patients with surgical outcomes, ${ }^{7,9}$ but patient satisfaction in this area was also not assessed in the present study. The second limitation is that the outcomes of PVCR for single-level hemivertebra were included in this study, and the mean angle of the preoperative main curve of $46.5^{\circ}$ indicated that the deformities in our patients were not severe overall. A progressive curve angle of greater than $25^{\circ}$ appeared to be the reason for an early intervention, especially for a 3-column resection, in some of the cases included here.

Posterior vertebral column resection has to include a complete circumferential excision of both bone and disc. Segmental resection or pedicle subtraction osteotomy fails to sufficiently correct the rigid fixed deformity in congenital scoliosis. Therefore, this deformity has to be corrected with PVCR because this procedure offers a greater extent of correction, particularly of kyphotic deformity. The PVCR approach enables sufficient shortening of the posterior column and helps achieve better bony union through improved bone-to-bone contact of the vertebral body even if the coronal curves are not severe. Our patients underwent excision of both the pedicle and vertebral body and of the discs above or below the hemivertebra at both sides, and these resections induced sufficient instability to correct the deformity. We note that even in cases of singlelevel hemivertebra, we excised discs along with the hemivertebra for complete correction because without the disc excision, the fixed deformity due to congenital scoliosis could not be corrected sufficiently.

It would be of interest to compare the success rate of correcting the deformity due to a single-level hemivertebra with that of correcting deformities involving more complex spinal abnormalities, but this question was not addressed in the present study. Another limitation is that the present study had only a small number of patients; therefore, additional studies with a larger number of patients will be needed to evaluate our results.

\section{Conclusions}

Posterior vertebral column resection is effective for treating patients with congenital scoliosis who are younger than 18 years. In these patients, PVCR and fusion with PSF achieved rigid fixation and satisfactory deformity correction that was maintained for more than 10 years. However, PVCR is a technically demanding procedure and entails risks for major complications and excessive blood loss.

\section{Acknowledgments}

We thank Dr. P. William Haake for English-language editing and Jiseok Jang for data collection for this paper.

\section{References}

1. Andrew T, Piggott H: Growth arrest for progressive scoliosis. Combined anterior and posterior fusion of the convexity. $\mathbf{J}$ Bone Joint Surg Br 67:193-197, 1985

2. Ayvaz M, Olgun ZD, Demirkiran HG, Alanay A, Yazici M: Posterior all-pedicle screw instrumentation combined with multiple chevron and concave rib osteotomies in the treatment of adolescent congenital kyphoscoliosis. Spine J 14:11-19, 2014

3. Boachie-Adjei O, Bradford DS: Vertebral column resection and arthrodesis for complex spinal deformities. J Spinal Disord 4:193-202, 1991

4. Bollini G, Docquier PL, Viehweger E, Launay F, Jouve JL: Lumbar hemivertebra resection. J Bone Joint Surg Am 88:1043-1052, 2006

5. Bollini G, Docquier PL, Viehweger E, Launay F, Jouve JL: Thoracolumbar hemivertebrae resection by double approach in a single procedure: long-term follow-up. Spine (Phila Pa 1976) 31:1745-1757, 2006

6. Bradford DS: Vertebral column resection. Orthop Trans 11:502, 1987 (Abstract)

7. Chang DG, Kim JH, Ha KY, Lee JS, Jang JS, Suk SI: Pos- 
terior hemivertebra resection and short segment fusion with pedicle screw fixation for congenital scoliosis in children younger than 10 years: greater than 7-year follow-up. Spine (Phila Pa 1976) 40:E484-E491, 2015

8. Chang DG, Kim JH, Kim SS, Lim DJ, Ha KY, Suk SI: How to improve shoulder balance in the surgical correction of double thoracic adolescent idiopathic scoliosis. Spine (Phila Pa 1976) 39:E1359-E1367, 2014

9. Chang DG, Suk SI, Kim JH, Ha KY, Na KH, Lee JH: Surgical outcomes by age at the time of surgery in the treatment of congenital scoliosis in children under age 10 years. Spine J 15:1783-1795, 2015

10. Crostelli M, Mazza O, Mariani M: Posterior approach lumbar and thoracolumbar hemivertebra resection in congenital scoliosis in children under 10 years of age: results with 3 years mean follow up. Eur Spine J 23:209-215, 2014

11. Dubousset J, Herring JA, Shufflebarger H: The crankshaft phenomenon. J Pediatr Orthop 9:541-550, 1989

12. Goldstein I, Makhoul IR, Weissman A, Drugan A: Hemivertebra: prenatal diagnosis, incidence and characteristics. Fetal Diagn Ther 20:121-126, 2005

13. Hedequist D, Emans J: Congenital scoliosis: a review and update. J Pediatr Orthop 27:106-116, 2007

14. Hedequist D, Emans J, Proctor M: Three rod technique facilitates hemivertebra wedge excision in young children through a posterior only approach. Spine (Phila Pa 1976) 34:E225E229, 2009

15. Jeszenszky D, Haschtmann D, Kleinstück FS, Sutter M, Eggspühler A, Weiss M, et al: Posterior vertebral column resection in early onset spinal deformities. Eur Spine J 23:198-208, 2014

16. Kim JH, Suk SI, Chung ER, Kim SS, Oh YM, Jeon JM, et al: Pedicle screw fixation in pediatric spinal deformity: results for patients under 10 years old. J Korean Orthop Assoc 40:583-590, 2005

17. Kim YJ, Lenke LG, Bridwell KH, Cho YS, Riew KD: Free hand pedicle screw placement in the thoracic spine: is it safe? Spine (Phila Pa 1976) 29:333-342, 2004

18. Lazar RD, Hall JE: Simultaneous anterior and posterior hemivertebra excision. Clin Orthop Relat Res (364):76-84, 1999

19. Leatherman KD: The management of rigid spinal curves. Clin Orthop Relat Res (93):215-224, 1973

20. Lenke LG, Newton PO, Sucato DJ, Shufflebarger HL, Emans JB, Sponseller PD, et al: Complications after 147 consecutive vertebral column resections for severe pediatric spinal deformity: a multicenter analysis. Spine (Phila Pa 1976) 38:119-132, 2013

21. Lenke LG, O'Leary PT, Bridwell KH, Sides BA, Koester LA, Blanke KM: Posterior vertebral column resection for severe pediatric deformity: minimum two-year follow-up of thirtyfive consecutive patients. Spine (Phila Pa 1976) 34:22132221, 2009

22. Lenke LG, Sides BA, Koester LA, Hensley M, Blanke KM: Vertebral column resection for the treatment of severe spinal deformity. Clin Orthop Relat Res 468:687-699, 2010

23. Li XF, Liu ZD, Hu GY, Chen B, Zhong GB, Zang WP, et al: Posterior unilateral pedicle subtraction osteotomy of hemivertebra for correction of the adolescent congenital spinal deformity. Spine J 11:111-118, 2011

24. MacLennan A: Scoliosis. BMJ 2:864-866, 1922

25. McMaster MJ, Ohtsuka K: The natural history of congenital scoliosis. A study of two hundred and fifty-one patients. J Bone Joint Surg Am 64:1128-1147, 1982

26. Modi HN, Suh SW, Fernandez H, Yang JH, Song HR: Accuracy and safety of pedicle screw placement in neuromuscular scoliosis with free-hand technique. Eur Spine J 17:16861696,2008

27. Nakamura H, Matsuda H, Konishi S, Yamano Y: Single-stage excision of hemivertebrae via the posterior approach alone for congenital spine deformity: follow-up period longer than ten years. Spine (Phila Pa 1976) 28:2132-2138, 2003

28. Nasca RJ, Stilling FH III, Stell HH: Progression of congenital scoliosis due to hemivertebrae and hemivertebrae with bars. J Bone Joint Surg Am 57:456-466, 1975

29. Olgun ZD, Demirkiran G, Ayvaz M, Karadeniz E, Yazici M: The effect of pedicle screw insertion at a young age on pedicle and canal development. Spine (Phila Pa 1976) 37:1778-1784, 2012

30. Ozturk C, Alanay A, Ganiyusufoglu K, Karadereler S, Ulusoy L, Hamzaoglu A: Short-term X-ray results of posterior vertebral column resection in severe congenital kyphosis, scoliosis, and kyphoscoliosis. Spine (Phila Pa 1976) 37:1054-1057, 2012

31. Ruf M, Harms J: Hemivertebra resection by a posterior approach: innovative operative technique and first results. Spine (Phila Pa 1976) 27:1116-1123, 2002

32. Ruf M, Harms J: Pedicle screws in 1- and 2-year-old children: technique, complications, and effect on further growth. Spine (Phila Pa 1976) 27:E460-E466, 2002

33. Ruf M, Harms J: Posterior hemivertebra resection with transpedicular instrumentation: early correction in children aged 1 to 6 years. Spine (Phila Pa 1976) 28:2132-2138, 2003

34. Ruf M, Jensen R, Letko L, Harms J: Hemivertebra resection and osteotomies in congenital spine deformity. Spine (Phila Pa 1976) 34:1791-1799, 2009

35. Suk SI, Chung ER, Kim JH, Kim SS, Lee JS, Choi WK: Posterior vertebral column resection for severe rigid scoliosis. Spine (Phila Pa 1976) 30:1682-1687, 2005

36. Suk SI, Chung ER, Lee SM, Lee JH, Kim SS, Kim JH: Posterior vertebral column resection in fixed lumbosacral deformity. Spine (Phila Pa 1976) 30:E703-E710, 2005

37. Suk SI, Kim JH, Kim WJ, Lee SM, Chung ER, Nah KH: Posterior vertebral column resection for severe spinal deformities. Spine (Phila Pa 1976) 27:2374-2382, 2002

38. Suk SI, Lee CK, Kim WJ, Chung YJ, Park YB: Segmental pedicle screw fixation in the treatment of thoracic idiopathic scoliosis. Spine (Phila Pa 1976) 20:1399-1405, 1995

39. Winter RB: Congenital scoliosis. Orthop Clin North Am 19:395-408, 1988

40. Winter RB, Moe JH, Lonstein JE: Posterior spinal arthrodesis for congenital scoliosis. An analysis of the cases of two hundred and ninety patients, five to nineteen years old. J Bone Joint Surg Am 66:1188-1197, 1984

41. Yang C, Zheng Z, Liu H, Wang J, Kim YJ, Cho S: Posterior vertebral column resection in spinal deformity: a systemic review. Eur Spine J [epub ahead of print], 2015

\section{Disclosures}

The authors report no conflict of interest concerning the materials or methods used in this study or the findings specified in this paper.

\section{Author Contributions}

Conception and design: Suk, Chang, Yang, Kim, Suh, Ha. Acquisition of data: Chang, Yang. Analysis and interpretation of data: Chang, Yang, Lee. Drafting the article: Chang, Yang. Critically revising the article: Suk, Chang, Yang. Reviewed submitted version of manuscript: Suk, Chang, Yang, Lee, Suh, Ha. Approved the final version of the manuscript on behalf of all authors: Suk. Administrative/technical/material support: Kim. Study supervision: Suk, Chang, Yang.

\section{Correspondence}

Se-Il Suk, Department of Orthopaedic Surgery, Inje University Sanggye Paik Hospital, 1342, Dongil-Ro, Nowon-Gu, Seoul 139707, Korea. email: seilsuk@unitel.co.kr. 